\title{
La recherche biomédicale aux Facultés Universitaires Notre-Dame-de-la-Paix
}

\author{
Robert Wattiaux, André Piront
}

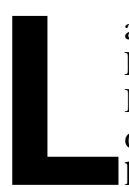

a recherche biomédicale aux Facultés Universitaires NotreDame-de-la-Paix a débuté dans les années 1960, lors de la création de la Faculté de Médecine. Elle s'est développée progressivement jusqu'à représenter actuellement une proportion importante de l'activité scientifique de l'institution. Une promotion marquée de cette recherche s'est produite à partir de 1973, du fait du développement à cette époque du département de biologie de la Faculté des Sciences.

\section{La recherche biomédicale à la Faculté de Médecine}

L'enseignement en Faculté de Médecine concerne uniquement les sciences précliniques. Les recherches sont par conséquent essentiellement du type fondamental et dépendent des départements d'histologie et d'embryologie, de physiologie et de biochimie.

- En Histologie, les travaux dirigés par les professeurs Leloup et Poumay, sont consacrés à certains aspects de la physiologie des kératinocytes épidermiques. Au cours de ces dernières années l'équipe d'histologie a développé un modèle d'étude des kératinocytes in vitro avec le Dr Pittelkow (Mayo Clinic), permettant de détecter l'induction des marqueurs précoces de la différenciation épider-

être évalués les rôles joués par différents facteurs de croissance de la famille de l'EGF(epidermal growth factor). Récemment, ce modèle a été mis à profit pour rechercher l'expression des molécules d'adhérence de la famille des intégrines.

- Au département de Physiologie, le groupe $\mathrm{du}$ professeur Goffinet a orienté ses recherches vers l'étude du développement cérébral. Il a étudié plus particulièrement la malformation génétique due à la mutation reeler de la souris, mutation qui perturbe une étape-clé du développement cérébral chez tous les mammifères. Après avoir réalisé une cartographie à haute résolution de la région chromosomique d'intérêt, le groupe du professeur Goffinet en même temps que d'autres équipes aux États-Unis et au Japon, a caractérisé le gène impliqué dans la mutation baptisé reelin. Les travaux de ce laboratoire se sont ensuite centrés sur l'étude de l'expression et du mécanisme d'action de la protéine induite par ce gène, la Reelin qui est sécrétée dans la matrice extracellulaire. Un autre groupe dirigé pendant de nombreuses années par le professeur De Schrijver, s'est intéressé à l'effet de l'hypoglycémie sur les astrocytes, cellules qui apparemment sont les premières affectées par la privation de glucose au niveau cérébral. Parmi les observations, signalons celles touchant le métabolisme du glutamate et de la glutamine, molécules essen- tielles dans la physiologie de l'astrocyte, montrant que l'activité de la glutamine synthétase était fortement diminuée lors de la privation de glucose. Tout récemment, sous l'impulsion du professeur Flamion, successeur du professeur De Schrijver, les recherches se sont davantage axées sur la physiologie rénale. Elles ont trait au rôle de la zone médullaire interne du rein dans l'équilibre hydroélectrolytique lors des épisodes de privation d'eau ou d'apport hydrique abondant ainsi que dans l'hypertension artérielle.

- Le département de Biochimie (professeurs Wattiaux-De Coninck, Jadot et Wattiaux) a une longue tradition de recherches dans le domaine des structures subcellulaires, en particulier des lysosomes. Mettant à profit les différentes méthodes fondées sur l'utilisation de la centrifugation et des marqueurs d'organites et, depuis quelques années, les techniques classiques de la biologie moléculaire, l'équipe de biochimie a réalisé de multiples travaux sur les propriétés des composants membranaires de la cellule et sur leur isolement. Citons à titre d'exemples ceux qui concernent l'effet de la pression hydrostatique engendrée par un champ centrifuge, les perturbants de densité, la purification des lysosomes, l'endocytose par les cellules sinusoïdales du foie. Quant aux lignes de recherches récentes, signalons l'étude de la structure de la matrice des lysosomes, 
de la capture et du cheminement de l'ADN exogène lors de la transfection, de certaines mutations dirigées sur la biosynthèse de la cathepsine $\mathrm{C}$, de la localisation subcellulaire du CFTR (cystic fibrosis transmembrane conductance regulator) muté.

\section{La recherche biomédicale} à la Faculté des Sciences

Ces recherches sont réalisées dans le département de Biologie de la Faculté des Sciences.

- En Biochimie cellulaire (professeurs Remacle et Raes), elles sont menées dans trois directions, chacune concernant la réponse cellulaire à un facteur externe, dans des situations normales et pathologiques. Est étudiée la réaction des fibroblastes au PDGF (platelet-derived growth factor) et à IL-1(interleukine-1). Les travaux sur la réaction cellulaire au PDGF sont focalisés sur l'analyse de l'inhibition de la cascade d'activation par les prostaglandines, les travaux sur les effets de IL-1 sont consacrés à l'analyse du mécanisme régulateur du NF-kB. Un autre groupe du laboratoire de Biochimie cellulaire travaille sur l'activation des cellules endothéliales par l'hypoxie. Ces recherches utilisent des cellules en culture provenant du cordon ombilical et des veines saphènes isolées. D'un point de vue médical, elles pourraient conduire à une meilleure connaissance de la physiopathologie des veines variqueuses. Enfin, est recherché l'effet du stress, en particulier le stress oxydatif sur le vieillissement cellulaire et ce, par une approche tant théorique (thermodynamique) qu'expérimentale.

- Dirigée, par le professeur Feytmans, les recherches du laboratoire de Biologie moléculaire structurale sont de nature théorique et expérimentale. Elles sont orientées vers l'étude de la structure des protéines et des relations entre la structure et la fonction de ces molécules. Elles portent sur les lactates déshydrogénases, la phosphofructokinase II du foie, la protéase de VIH, la porine Omzb de Brucella melitensis. Grâce à des méthodes d'ingénierie par mutagenèse dirigée, elles ont conduit récemment à des résultats intéres- sants sur la phosphofructokinase II hépatique.

- Le laboratoire de Génétique moléculaire suit deux axes de recherches principaux, utilisant la levure comme système modèle. L'un porte sur la signification de deux adénines diméthylées situées à l'extrémité 3' des ARN de la petite sous-unité ribosomique. Les travaux ont montré que le gène DIMI de Saccharomyces cerevisiae était essentiel et que la protéine DimIp jouait un rôle dans la maturation du pré-ARNt. L'autre direction de recherches s'intéresse à la protéine régulatrice MigIp chez Luyveromyces lactis, en particulier aux relations structure-fonction de cette protéine.

Nous voudrions pour terminer mettre l'accent sur le fait que la recherche biomédicale aux Facultés Universitaires Notre-Dame-de-la-Paix est favorisée grâce à de nombreuses relations des chercheurs de l'institution avec des laboratoires d'autres universités belges et étrangères et qu'elle est soutenue par divers fonds extérieurs

\section{Robert Wattiaux}

Professeur émérite.

\section{André Piront}

Doyen de la faculté de médecine. Facultés Universitaires Notre-Dame-de-la-Paix, Faculté de médecine, Bureau du doyen, rue de Bruxelles, 61, B-5000 Namur, Belgique.

\section{TIRÉS À PART}

A. Piront.

\section{ACIDES GRAS}

et

NUTRITION

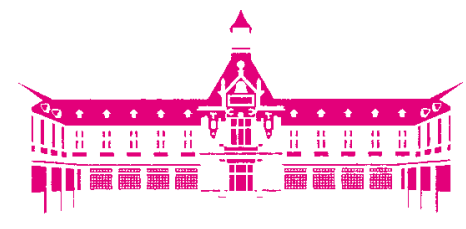

RENNES

\section{4-15 mai 1998}

École Nationale Supérieure

Agronomique 